\title{
Quantification of Cerebral Grey and White Matter Asymmetry from MRI
}

\author{
Frederik Maes ${ }^{1}$, Koen Van Leemput ${ }^{1}$, Lynn E. DeLisi ${ }^{2}$, \\ Dirk Vandermeulen ${ }^{1}$, and Paul Suetens ${ }^{1}$ \\ 1 Medical Image Computing (Radiology-ESAT) \\ Katholieke Universiteit Leuven \\ UZ Gasthuisberg, Herestraat 49, B-3000 Leuven, Belgium \\ Frederik.Maes@uz.kuleuven.ac.be \\ 2 SUNY Stony Brook, Department of Psychiatry \\ Health Sciences Center, Stony Brook, N.Y. 11794, USA \\ ldelisi@ccmail.sunysb.edu
}

\begin{abstract}
We present a completely automated procedure for measuring left and right hemispheric asymmetry in cerebral grey and white matter volumes from MR images using a chain of state-of-the-art image analysis algorithms. After bias correction and tissue classification, left and right hemispheres are separated by non-rigid registration to a template image in which both hemispheres have been carefully segmented. Volume renderings of each hemisphere separately demonstrate the high quality of the resulting segmentations. Because all steps in the procedure are completely automated and do not require user specified parameters, the results are highly reproducible and consistent. We present quantitative results obtained from a database of MR images of 40 schizophrenic patients and 31 normal controls.
\end{abstract}

\section{Introduction}

Several studies have reported morphological differences in the brains of schizophrenic patients when compared to normal controls [1], including decreased or reversed cerebral asymmetries. Current MR imaging techniques allow to acquire $3 \mathrm{D}$ images of the brain with high resolution and signal to noise ratio, which makes it feasible to measure brain morphology in vivo. But slice by slice manual delineation of structures of interest in 3D images by a human expert is tedious and usually subject to large inter- and intra-observer variability and poor reproducibility. This deteriorates the statistical significance of the reported morphological findings and may also explain some inconsistencies across studies. Moreover, in longitudinal studies aiming at detecting morphological changes over time, the quality of historical images is generally inferior to what is possible today. Hence the need for automated computer methods for image based morphometry which yield accurate, consistent and reproducible results and which are robust against imaging imperfections in historical data.

In this paper, we present a method for quantifying cerebral grey and white matter asymmetry from MR images. The method consists of bias field correction, 
tissue segmentation and atlas-based separation of left and right hemispheres and relies on state-of-the-art algorithms for rigid and non-rigid image registration and intensity-based voxel classification. Because all steps in the procedure are completely automated and do not require interactive intervention or user specified parameters, the results are highly reproducible and consistent. We present quantitative results obtained from a database of MR images of schizophrenic patients and normal controls within the scope of the European BIOMORPH Project [2].

\section{Method}

Bias correction and tissue classification White and grey matter are segmented after bias correction by the intensity-based tissue classification algorithm of Van Leemput [3]. The method models the single or multi-channel MR signal as the realization of a random process, the intensities of each channel within each tissue class being drawn from a Gaussian distribution, but which is corrupted by a polynomial intensity inhomogeneity or bias field. The algorithm maximizes the a posteriori likelihood of the model parameters given the data by an Expectation-Maximization procedure, iteratively alternating between tissue classification, class intensity distribution estimation and bias field estimation. The method, which is fully automated, is initialized with a priori spatial distribution maps for grey matter, white matter and csf obtained from a digital brain atlas that is distributed with the SPM software package [4]. The atlas image is normalised to the space of the study image by an affine transformation which is computed automatically by maximizing mutual information between both images [5]. Only voxels to which the atlas assigns a non-zero a priori brain tissue probability are considered during segmentation.

Left/right hemispheric separation Separation of the computed white and grey matter probability maps into left and right halves is achieved by non-rigid registration of the study image to a template MR image in which left and right hemispheres have been carefully segmented. This image and the segmentations were retrieved from the IBSR [6]. The delineations of left and right hemispheres were transformed into binary label images, which were subsequently edited by morphological operations to match the brain envelope rather than the individual gyri and sulci to be more robust against differences in local cortical topology (figure 1). The template image is matched to the study image by a combination of affine and locally non-rigid transformations, using the non-rigid intensity-based matching algorithm of Thirion [7]. The same transformation is then applied to the label images, which yields matched outlines of both hemispheres that allow to separate left and right halves in the tissue segmentations and, at the same time, to remove non-relevant structures such as the cerebellum and brain stem.

White and grey matter volumetry Finally, volumes for grey and white matter for each brain half separately are computed by integrating the corresponding probability maps within the brain regions of interest defined by the matched template image. 


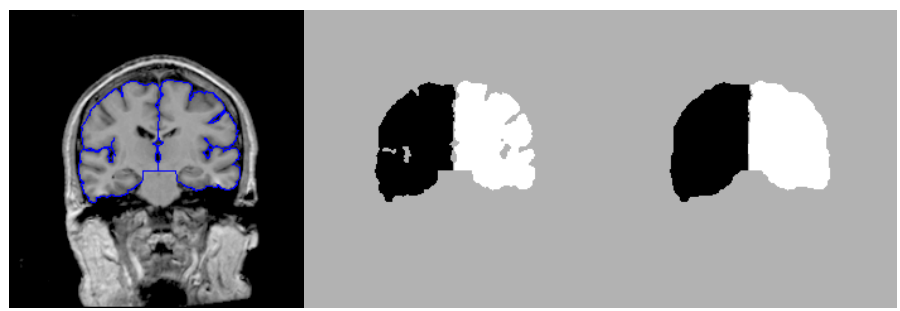

Fig. 1. Template of left and right hemispheres. Left: original MR image slice in which left and right hemispheres have been carefully segmented manually by a trained expert. Middle: conversion of the original contours into a binary template image. Right: the template image is edited to match the brain envelope rather than the individual gyri and sulci to be more robust against differences in local cortical topology.

\section{Results}

\subsection{D MPRAGE}

Figure 2 illustrates the performance of the method on high quality high resolution data, consisting of a single sagittal T1-weighted image (Siemens Vision 1.5 T, 3D MPRAGE, $256 \times 256$ matrix, $1.25 \mathrm{~mm}$ slice thickness, 128 slices, $\mathrm{FOV}=256 \mathrm{~mm}, \mathrm{TR}=11.4 \mathrm{~ms}, \mathrm{TE}=4.4 \mathrm{~ms}$ ) with good contrast between grey matter, white matter and the tissues surrounding the brain. The grey and white matter segmentation maps obtained from the original images are correctly split in separate maps for left and right hemispheres by non-rigid registration with the labeled template image. The high quality of the segmentations and of the left/right separation is clearly illustrated by volume renderings of the grey and white matter maps of each brain half.

\subsection{BIOMORPH Schizophrenia Data}

The method was applied to a database of MR images, available within the BIOMORPH project [2], that was collected at SUNY, Stony Brook, N.Y, USA, as part of a prospective follow-up study of brain morphology in schizophrenic patients. Left and right white and grey matter volumes were segmented from a single coronal T1-weighted MR scan (GE Signa 1.5 T, 3D GRASS, $256 \times 256$ matrix, $1.5 \mathrm{~mm}$ slice thickness, 124 slices, FOV $=240 \mathrm{~mm}$, TR $=24 \mathrm{~ms}$, $\mathrm{TE}=5 \mathrm{~ms}$ ) for a group of 40 patients (26 males, 14 females, mean age was $27.7 \pm 6.0)$ and 31 controls (18 males, 13 females, mean age was $30.6 \pm 7.9)$.

A typical segmentation result in shown in figure 3. Normalising the multiplicative bias field to a mean value of 1 , bias correction factors within the brain region typically range from 0.7 to 1.4 with a standard deviation of 0.06 , which is clearly not negligible. The apparant symmetry of the resulting segmentations illustrates that the segmentation procedure is indeed capable of correcting for 

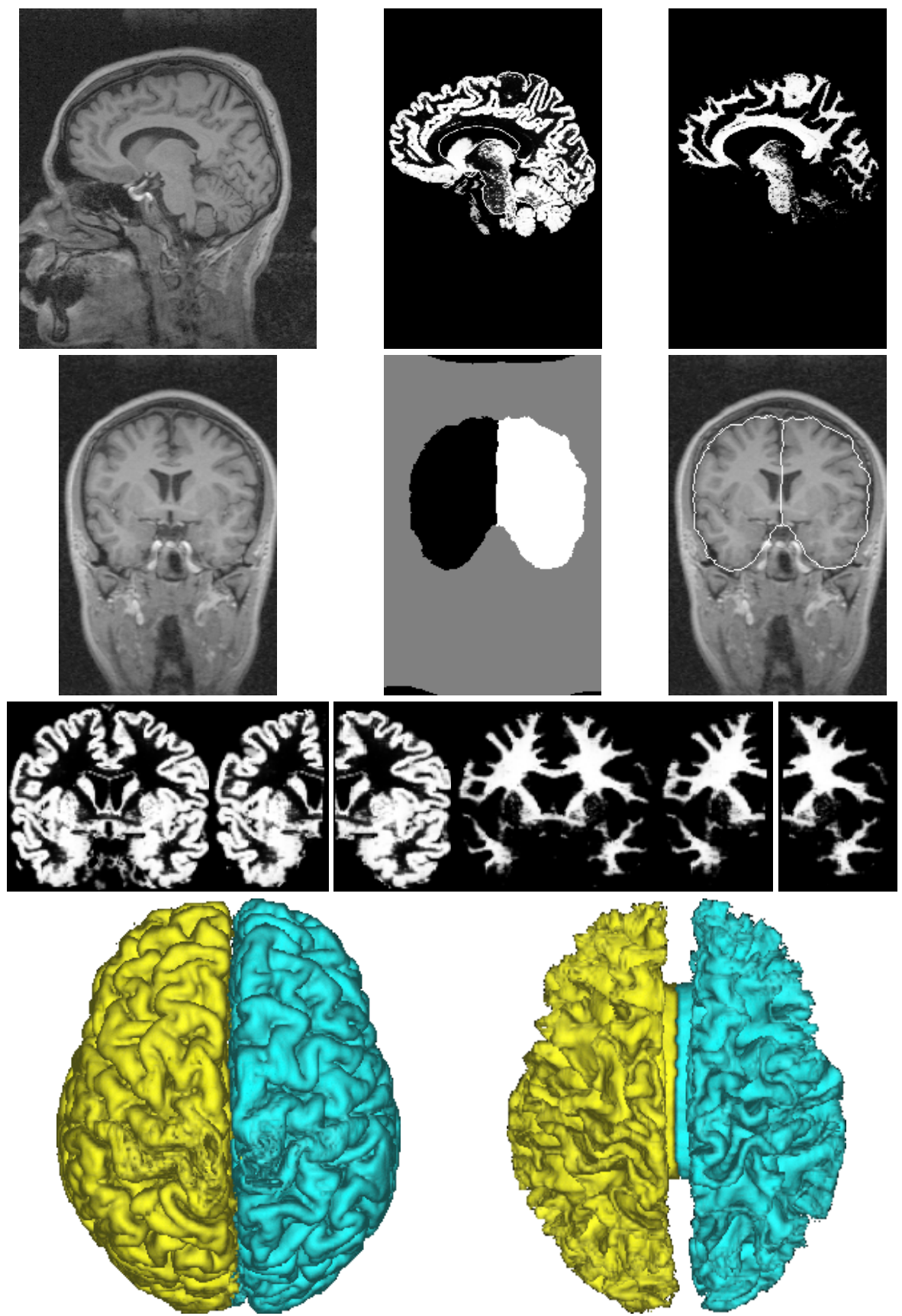

Fig. 2. Top: original sagittal MPRAGE image and grey and white matter segmentations. Middle: coronal cross section with matched labeled template and grey and white matter segmentations before and after hemispheric separation. Bottom: volume renderings of grey and white matter segmentation maps for each hemisphere separately. 
significant bias. Note that some voxels outside of the brain are erroneously classified as grey or, to a lesser extent, white matter. These voxels were assigned a non-zero a priori probability for these classes by the atlas used to initialize the segmentation process and because their intensity in these images is similar to those of the true grey or white matter voxels, the classification algorithm, which treats each pixel independently, is not able to discriminate between them.
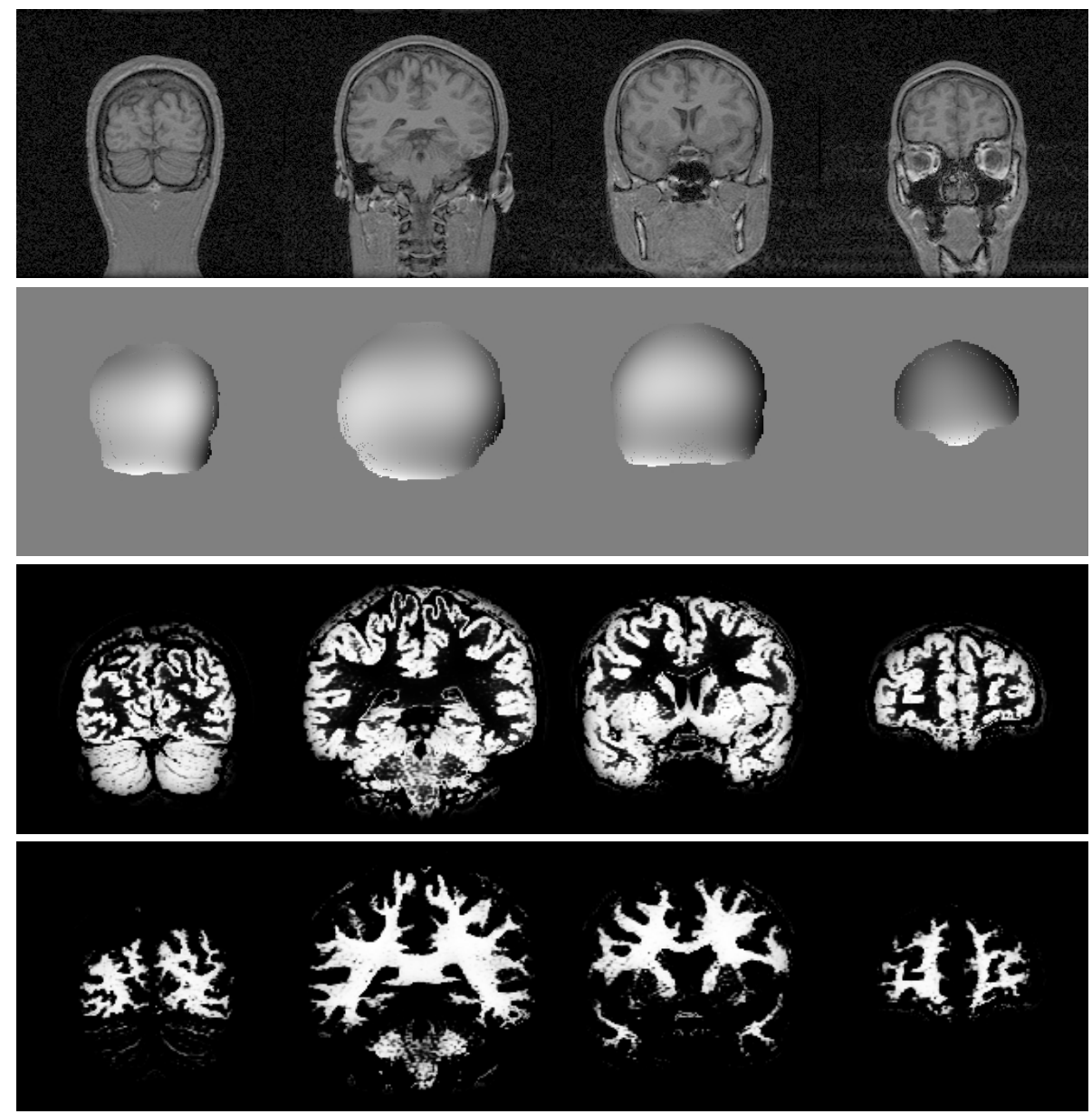

Fig. 3. Original T1-weighted image, estimated bias field and resulting grey and white matter segmentation for a typical BIOMORPH schizophrenia data set.

Grey and white matter segmentations for each hemisphere separately obtained after non-rigid registration of the labeled template image are shown in figure 4. Inspection of the results shows that separation of both brain halves, aswell as of cerebellum and brain stem, is generally excellent. This is also illus- 
trated by volume renderings of the grey and white matter probability maps. The non-rigidly matched template image provides a more precise brain mask than the one used for segmentation, such that most of the wrongly classified non-brain voxels are removed when separating both brain halves.

\subsection{Volume Measurements}

Grey and white matter volume measurements obtained from the BIOMORPH database of patients and controls are summarised in figure 5 and table 1 . Volumes are obviously larger in males than in females and both grey matter and white matter show the tendency to be decreased in patients versus controls. We also found a slight difference between grey matter volumes left and right (about 2.5 $\mathrm{cm}^{3}$ or $0.8 \%$ on average), both in schizophrenics and controls. Statistical analysis of these results and correlation with diagnosis, age, sex and dexterity is currently in progress.

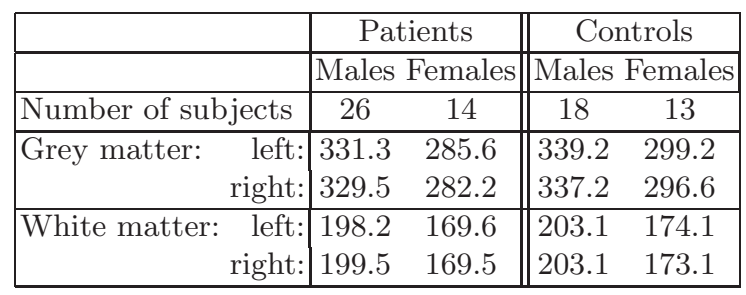

Table 1. Mean left and right cerebral grey and white matter volumes (in $\mathrm{cm}^{3}$ ) for the BIOMORPH database of patients and controls for males and females separately.

\section{Discussion}

Our grey and white matter segmentation method has been validated on simulated MR images with known ground truth and on real MR images by comparison with expert delineations [12]. We found that the automated and the manual segmentations consistently show excellent overlap (average overlap metric [13] values of $95 \%, 83 \%$ and $82 \%$ for total brain, grey matter and white matter respectively) and that differences mainly occur at the white matter/grey matter and grey matter/csf interfaces, which can be attributed to partial volume effects on the one hand and to the tendency of the automated method to more meticulously follow tissue borders than the manual tracer on the other hand. For 2 patients and 3 controls in the BIOMORPH database, two scans were available at two time points about one year apart. The mean difference between corresponding measurements at different time points was $1 \%$ for controls and $4 \%$ for 

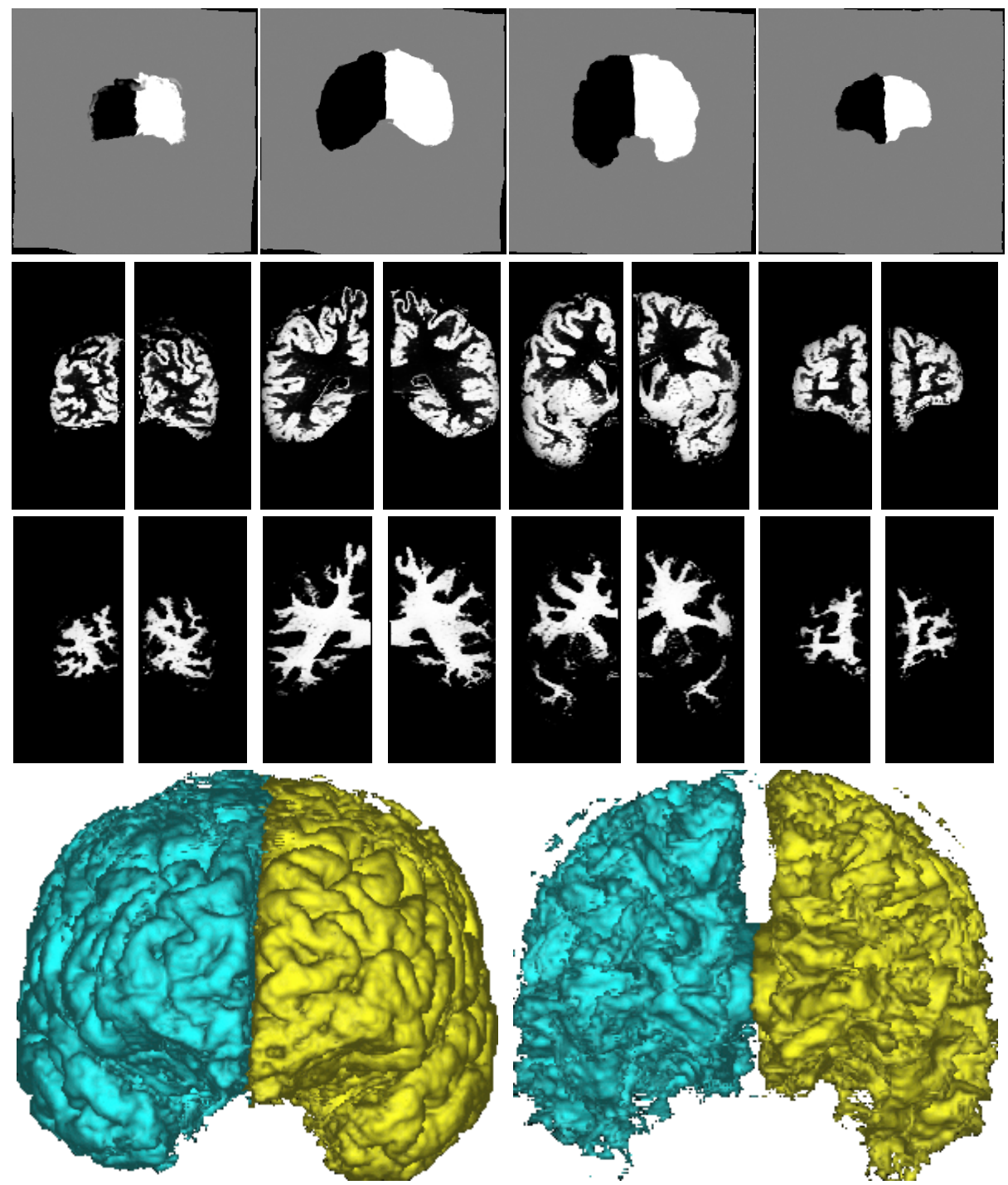

Fig. 4. After non-rigid registration with the labeled template image (top), the original grey and white matter segmentations can be separated into left and right alves and non-relevant structures, such as the cerebellum and the brain stem, can be removed. 


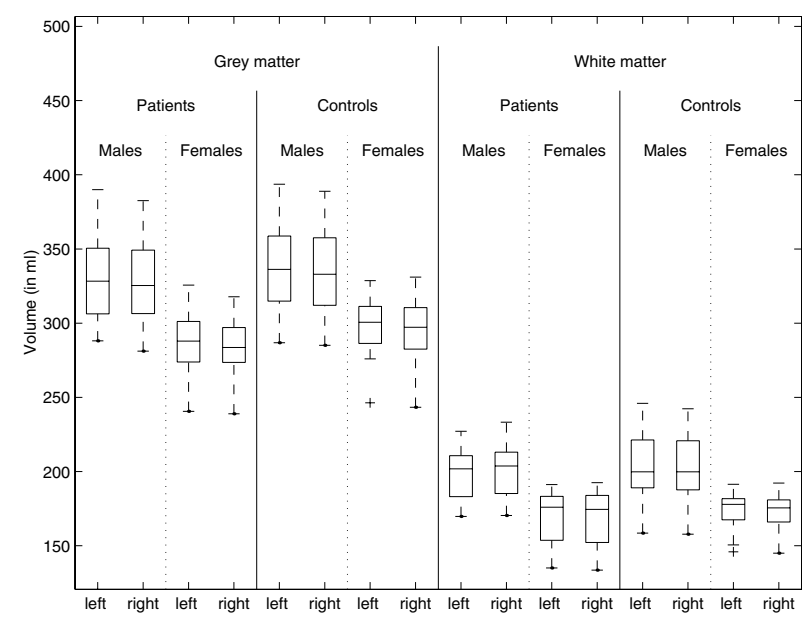

Fig. 5. Left and right cerebral grey and white matter volumes (in $\mathrm{cm}^{3}$ ) for the BIOMORPH database of patients and controls for males and females separately.

patients. While real changes over time may be neglected in controls but can not be excluded in patients, we estimate the reproducibility of the method to be about $1 \%$.

Various authors have presented various techniques to separate the brain hemispheres by the so-called mid-sagittal plane, defined as the plane that best fits the inter-hemispheric fissure of the brain [8] or as the plane that maximizes similarity between the image and its reflection relative to this plane $[9,10]$. The advantage of our approach of intensity-driven non-rigid registration to a labeled template image is that it doesn't assume the boundary between both hemispheres to be planar. Dawant [11] used exactly the same algorithm for automated atlas-based segmentation of internal brain structures and found that, for large scale structures such as the brain or the cerebellum, the resulting contours are virtually indistinguishable from outlines drawn by an experienced radiologist. The same technique can be applied to analyse asymmetry in subregions such as the frontal or temporal lobe.

Our approach assumes that differences in (dis)symmetry between the template image and the study images are completely resolved by the non-rigid registration. If this is not the case, bias may be introduced in the measurements due to the (dis)symmetry of the template image itself, which we derived from a single subject's scan. Such bias could be detected by doing the measurements again with the left/right axis in the template image reversed. Alternatively, an unbiased virtual brain template could be created by averaging images of several individuals and their reflected versions and delineating the average image.

Disturbing in the analysis of the BIOMORPH data is the influence of nonbrain pixels that are falsely classified as grey matter, due to a lack of contrast between true grey matter and some non-brain tissues in these images. If additional 
MR channels were available that would allow to differentiate between these tissues, the problem would be solved by multi-channel segmentation. Incorporating spatial constraints in the classification, such as prohibiting grey matter pixels to be surrounded by non-brain pixels [12], improved the single-channel classification of the BIOMORPH data slightly, but still showed important misclassifications. While non-rigid registration to the template image allows to exclude many of the misclassified non-brain pixels, the same non-rigid registration approach could be used to generate much sharper a priori tissue probability maps than the ones we are using now to initialize the segmentation process. We expect that nonrigid registration of such an atlas to the study images will yield a better brain mask and improved segmentations. Alternatively, the problem may be solved by straightforward morphological processing of the segmentation maps, including erosion/dilation and largest component selection.

\section{Summary and Conclusions}

We presented a completely automated procedure for consistent and reproducible quantification of left and right cerebral white and grey matter volumes from MR images, which combines state-of-the-art algorithms for tissue classification and atlas-based segmentation. The method has been applied on a database of high resolution images of schizophrenic patients and normal controls. Further work will focus on reducing the influence of falsely classified non-brain pixels and on extending the approach to smaller subregions in the brain.

\section{Acknowledgments}

This work was supported by the EC-funded BIOMORPH project 95-0845 (Development and Validation of Techniques for Brain Morphometry), a collaboration between the Universities of Kent and Oxford (UK), ETH Zürich (Switzerland), INRIA Sophia Antipolis (France) and KU Leuven (Belgium), and by the Research Fund KU Leuven GOA/99/05 (Variability in Human Shape and Speech). The template image and its manual segmentation was provided by the Center for Morphometric Analysis at Massachusetts General Hospital, Charlestown, MA, USA, and is available at http://neuro-www.mgh.harvard.edu/cma/ibsr. The non-rigid registration software was provided by Jean-Philippe Thirion.

\section{References}

1. L.E. DeLisi, W. Tew, S. Xie, A.L. Hoff, M. Sakuma, M. Kushner, G. Lee, K. Shedlack, A.M. Smith, and R. Grimson. A prospective follow-up study of brain morphology and cognition in first-epsiode schizophrenic patients: preliminary findings. Biol Psychiatry, 38:349-360, 1995. 348

2. Development and validation of techniques for brain morphometry (BIOMORPH). Biomed Project No. 95-0845. Partners in the BIOMORPH project are: University of Kent at Canterbury, Kent, UK; ETH, Zürich, Switzerland; University of Oxford, Oxford, UK; INRIA, Nice, France; K.U. Leuven, Leuven, Belgium. 349, 350 
3. K. Van Leemput, F. Maes, D. Vandermeulen, and P. Suetens. Automatic segmentation of brain tissues and MR bias field correction using a digital brain atlas. In W.M. Wells, A. Colchester, and S. Delp, editors, Medical Image Computing and Computer-Assisted Intervention (MICCAI'98), volume 1496 of Lecture Notes in Computer Science, pages 1222-1229, Cambridge, MA, USA, October 1998. Springer. 349

4. J. Ashburner, K. Friston, A. Holmes, and J. Poline. Statistical Parametric Mapping. The Wellcome Department of Cognitive Neurology, University College London. 349

5. F. Maes, A. Collignon, D. Vandermeulen, G. Marchal, and P. Suetens. Multimodality image registration by maximization of mutual information. IEEE Transactions on Medical Imaging, 16(2):187-198, April 1997. 349

6. Internet Brain Segmentation Repository (IBSR). Maintained by Andrew Worth at the Center for Morphometric Analysis, Massachusetts General Hospital, Charlestown, MA, USA. http://neuro-www.mgh.harvard.edu/cma/ibsr. 349

7. J.-P. Thirion. Fast non-rigid matching of 3D medical images. In Medical Robotics and Computer Aided Surgery (MRCAS'95), pages 47-54, Baltimore, MD, USA, November 1995. 349

8. P. Marais, R. Guillemaud, M. Sakuma, A. Zisserman, and M. Brady. Visualising cerebral asymmetry. In K.H. Höhne and R. Kikinis, editors, Visualization in Biomedical Computing, volume 1131 of Lecture Notes in Computer Science, pages 411-416, Hamburg, Germany, September 1996. Springer. 355

9. Y. Liu, R.T. Collins, and W.E. Rothfus. Automatic bilateral symmetry (midsagittal) plane extraction from pathological 3D neuroradiological images. In K.M. Hanson, editor, Medical Imaging 1998: Image Processing, volume 3338 of Proc. SPIE, pages 1528-1539, San Diego, CA, USA, February 1998. 355

10. S. Prima, J.-P. Thirion, G. Subsol, and N. Roberts. Automatic analysis of normal brain dissymmetry of males and females in MR images. In W.M. Wells, A. Colchester, and S. Delp, editors, Medical Image Computing and Computer-Assisted Intervention (MICCAI'98), volume 1496 of Lecture Notes in Computer Science, pages 770-779, Cambridge, MA, USA, October 1998. Springer. 355

11. B.M. Dawant, J.-P. Thirion, F. Maes, D. Vandermeulen, and P. Demaerel. Automatic 3D segmentation of internal structures of the head in MR images using a combination of similarity and free-form transformations. In K.M. Hanson, editor, Medical Imaging 1998: Image Processing, volume 3338 of Proc. SPIE, pages 1528-1539, San Diego, CA, USA, February 1998. 355

12. K. Van Leemput, F. Maes, D. Vandermeulen, and P. Suetens. Automated modelbased tissue classification of MR images of the brain. Submitted to IEEE Trans. Medical Imaging, January 1999. 353, 356

13. A. Zijdenbos, B. M. Dawant, R. A. Margolin, and A. C. Palmer. Morphometric analysis of white matter lesions in MR images: Method and validation. IEEE Transactions on Medical Imaging, 13(4):716-724, December 1994. 353 